\title{
Effects of the Salt-Processing Method on the Pharmacokinetics and Tissue Distribution of Orally Administered Morinda officinalis How. Extract
}

\author{
Ji Shi $(\mathbb{D}$, Xiaohang Ren, Jia Wang, Xiaofeng Wei, Bonan Liu, and Tianzhu Jia \\ School of Pharmacy, Liaoning University of Traditional Chinese Medicine, Dalian 116600, China \\ Correspondence should be addressed to Tianzhu Jia; tianzhujia0411@163.com
}

Received 10 October 2019; Revised 2 December 2019; Accepted 11 December 2019; Published 11 February 2020

Academic Editor: Hana Sklenarova

Copyright (C) 2020 Ji Shi et al. This is an open access article distributed under the Creative Commons Attribution License, which permits unrestricted use, distribution, and reproduction in any medium, provided the original work is properly cited.

\begin{abstract}
Salt processing, which involves steaming with salt water, directs herbs into the kidney channel. After being salt processed, kidney invigorating effects occur. However, the underlying mechanism of this method remains elusive. The compounds monotropein, rubiadin, and rubiadin 1-methyl ether are the major effective components of Morinda officinalis How. To clarify the pharmacokinetics and tissue distribution of these three compounds, we employed liquid chromatography-tandem mass spectrometry (UPLC-MS/MS) to determine the contents of the three components in rat plasma and tissues. Separation was achieved on an Acquity UPLC HSS T3 column $(100 \mathrm{~mm} \times 2.1 \mathrm{~mm}, 1.8 \mu \mathrm{m}$, Waters). Formic acid aqueous solution $(0.1 \%$; A) and acetonitrile (containing $0.1 \%$ formic acid; B) were used as the mobile phase system with a programmed elution of $0 \sim 5$ min with $70 \%$ A and then $5 \sim 7$ min with $60 \% \mathrm{~A}$. All analytes were measured with optimized multiple reaction monitoring (MRM) in negative ion mode. Geniposide and 1,8-dihydroxyanthraquinone were used as the internal standards (IS). The linear ranges were 1.2 190, 1.3 510, and $0.047 \sim 37.5 \mu \mathrm{g} / \mathrm{mL}$, respectively. Compared with the Morinda officinalis without wood (MO) group, the $\mathrm{C}_{\mathrm{ma}} \mathrm{x}$ and $\mathrm{AUC}_{0-\mathrm{t}}$ parameters of rubiadin and rubiadin 1-methyl ether elevated remarkably for the salt-processed Morinda officinalis (SMO) groups, which indicates that steaming by salt could increase the bioavailability of rubiadin and rubiadin 1-methyl ether. The $T_{\max }$ for monotropein is shorter $(0.5 \mathrm{~h})$ in SMO groups than that in MO group, which means that monotropein was quickly absorbed in the SMO extract. Moreover, the contents of three compounds in the small intestine were the highest.
\end{abstract}

\section{Introduction}

Morinda officinalis is a commonly used traditional Chinese medicines (TCMs) [1], which has been used in China for many years. People use it as a tonic or tonifying kidney product to protect against and cure depression, rheumatoid arthritis, osteoporosis, and kidney-yang deficiency syndrome [2-6]. Its main active components include oligosaccharides, polysaccharides, iridoid glycosides, and anthraquinones [7-9].

Chinese medicinal herb processing is a unique pharmaceutical technique, which can turn Chinese medicinal materials into decoction pieces using processing methods like stir-frying, steaming, and frying with sand or wheat bran [10]. Decocting pieces are the main form prescribed in TCM clinics [1]. The pharmacological actions and energy properties (nature, flavor, and channel tropism) might be changed after processing, side effects and disagreeable odors can be eliminated. It is essential to use the proper processing method to ensure the quality and safety of traditional Chinese medicine decocting pieces. Steaming or stir-frying with salt can change the activity direction of Chinese herbal medicines and can also improve their efficacy [11-14].

The commercially available products of Morinda officinalis in herbal markets are M. officinalis without wood (MO) and salt-processed products (SMO). It was revealed that $\mathrm{MO}$ extract exerts tonifying kidney and supporting yang effects by regulating the functions of the hypothalamus-pituitaryadrenal axis $[15,16]$. The medicinal efficacy of MO and SMO is different because of their different processing methods. After being salt processed, the effects of strengthening kidney-yang can be dramatically enhanced. The chemical 
composition changes during processing, for example, glycosides can be hydrolyzed into aglycone or converted into other constituents, the contents of toxic ingredients can be decreased or converted into other constituents [17, 18].

Monotropein, which possess anti-inflammatory, analgesia, and antiosteoporotic activities, is the main iridoid glycoside isolated from M. officinalis $[19,20]$. Anthraquinones, especially alizarin-anthraquinones, like rubiadin and rubiadin 1-methyl ether, can inhibit osteoclastic bone resorption in vitro and invigorate the kidney-yang [21-24]. Therefore, in the present study, we chose monotropein, rubiadin, and rubiadin 1-methyl ether as the representative compounds to explain the effects of the different processing methods on the pharmacokinetics of M. officinalis.

There have been reports on the pharmacokinetics or tissue distribution of iridoids and oligosaccharides using HPC-DAD and LC-MS [25-28]. However, the abovementioned studies paid more attention to pure iridoid, inulin-type fructo-oligosaccharides and neglected interactions among iridoids and other compounds. There still has been no report about the pharmacokinetics and tissue distribution characteristics of alizarin-anthraquinones in $M$. officinalis. Also, the effect of processing methods on the pharmacokinetics, bioavailability, and tissue distribution characteristics of iridoids and anthraquinones has barely been reported.

In this study, we developed a selective and sensitive UPLC-MS/MS method for simultaneous determination of monotropein, rubiadin, and rubiadin 1-methyl ether in rat plasma and tissues. The analysis method was successfully applied to the pharmacokinetics study of MO and SMO extracts.

\section{Materials and Methods}

2.1. Materials and Chemicals. M. officinalis were collected from Gaoliang County, Guangdong Province, and identified by Prof. Feng Li of the Liaoning University of Traditional Chinese Medicine. For reference substance, rubiadin (J0111AS), rubiadin 1-methyl ether (J0307AS), monotropein (00605AS) were purchased from Meilun Biological Technology Co. Ltd. (Shanghai, China). The internal standards (ISs) geniposide (1203A023) and 1,8-dihydroxyanthraquinone (100398-200701) were provided by the National Institute for Food and Drug Control (Beijing, China).

Merck KGaA (Darmstadt, Germany) supplied LC-MSgrade formic acid and acetonitrile (HPLC-grade). The ultrapure water was generated with a Milli-Q water purification system (18.2 M $\Omega$, Millipore, Billerica, USA). Other reagents were of analytical grade from Tianjin Kermol Chemical Reagent Co., Ltd. (Tianjin, China) and Waters Xevo TQD Mass Spectrometer (Massachusetts, USA).

2.2. Animals. Sprague-Dawley (SD) rats $(200 \pm 20$ g, weight $)$ were purchased from Changsheng Biotechnology Co. Ltd. (License No. SCXK (Liao) 2015-0001, Benxi, Liaoning Province). The experimental animals were kept at $25 \pm 2^{\circ} \mathrm{C}$ and $60 \% \pm 10 \%$ humidity with a $12 \mathrm{~h}$ light/dark cycle. Water and chow were provided ad libitum. All animal pharmacological experiments followed the ethical regulations of the Liaoning University of Traditional Chinese Medicine.

2.3. LC-MS Analytical Conditions. For LC-MS/MS analysis, data acquisition and instrument control were performed using MassLynx 4.1 software (Waters Corp., Milford, MA, USA). The analysis column was an Acquity UPLC HSS T3 column $(100 \mathrm{~mm} \times 2.1 \mathrm{~mm}, 1.8 \mu \mathrm{m}$, Waters $)$ with a temperature of $40^{\circ} \mathrm{C}$. The mobile phase was acetonitrile containing $0.1 \%$ formic acid (A) and $0.1 \%$ formic acid aqueous solution (B). The elution gradient was $0.00-0.50 \mathrm{~min}$ with $2 \% \mathrm{~B} ; 0.51-5.00 \mathrm{~min}$ with $2 \%-30 \% \mathrm{~B}$; and $5.01-7.00 \mathrm{~min}$ with $40 \% \mathrm{~B}$. The flow rate was $0.2 \mathrm{~mL} / \mathrm{min}$.

The Waters triple quadruple mass spectrometer (Xevo TQD, Waters Corp., Milford, MA, USA) equipped with an electrospray ionization source (ESI) was used in negative ion mode. The desolvation gas was nitrogen with a flow rate of $500 \mathrm{~L} / \mathrm{h}$ at a temperature of $250^{\circ} \mathrm{C}$. All detected compounds were measured in multiple reaction monitoring (MRM) mode. Figure 1 shows the chemical structures of the three analytes and the internal standards.

The precursor and product ion pairs for MRM were $\mathrm{m} / \mathrm{z}$ $389.01 \longrightarrow 191.01$ for monotropein (collision energy $15 \mathrm{eV}$ ); $\mathrm{m} / \mathrm{z} 253.05 \longrightarrow 195.21$ for rubiadin (collision energy $40 \mathrm{eV}$ ); $\mathrm{m} / \mathrm{z} 267.07 \longrightarrow 252.04$ for rubiadin 1-methyl ether (collision energy $18 \mathrm{eV}$ ); $\mathrm{m} / \mathrm{z} 387.03 \longrightarrow 225.03$ for IS geniposide (collision energy $8 \mathrm{eV}$ ); and $\mathrm{m} / z 238.94 \longrightarrow 166.99$ for IS 1,8 dihydroxyanthraquinone (collision energy $35 \mathrm{eV}$ ).

2.4. Preparation of Reference Substances. Monotropein, rubiadin, and rubiadin 1-methyl ether were dissolved in methanol to prepare a stock solution with a concentration of $0.5 \mathrm{mg} / \mathrm{mL}$. The stock solution was diluted with methanol to get the appropriate concentrations for the working standard solutions. The IS stock solution $(1 \mathrm{mg} / \mathrm{mL})$ and IS working solution $(200 \mathrm{ng} / \mathrm{mL})$ were also prepared in methanol, as described above. All prepared solutions were stored at $4^{\circ} \mathrm{C}$ before use.

The preparation of calibration standards was done as follows: The appropriate working solution was spiked into blank plasma or tissues to obtain concentrations of $75 \sim 0.047 \mu \mathrm{g} / \mathrm{mL}$ for monotropein, $396 \sim 1.54 \mu \mathrm{g} / \mathrm{mL}$ for rubiadin, and $596 \sim 1.16 \mu \mathrm{g} / \mathrm{mL}$ for rubiadin 1 -methyl ether. The QC concentrations for monotropein were $0.14 \mu \mathrm{g} / \mathrm{mL}$, $2 \mu \mathrm{g} / \mathrm{mL}$, and $28.1 \mu \mathrm{g} / \mathrm{mL}$; for rubiadin, they were $1.6 \mu \mathrm{g} / \mathrm{mL}$, $50.6 \mu \mathrm{g} / \mathrm{mL}$, and $712.5 \mu \mathrm{g} / \mathrm{mL}$; and for rubiadin 1-methyl ether, they were $3.9 \mu \mathrm{g} / \mathrm{mL}, 54.6 \mu \mathrm{g} / \mathrm{mL}$, and $765 \mu \mathrm{g} / \mathrm{mL}$. The QC samples used for the recovery, matrix effect, intra- and interday accuracy, precision, and stability studies were prepared in the same way as that of the calibration standards. The solutions were stored for one week at $-20^{\circ} \mathrm{C}$.

2.5. Preparation of MO and SMO Extract. We obtained the products of $M$. officinalis without wood and salt-processed M. officinalis by following processing methods. 
<smiles>Cc1c(O)cc2c(c1O)C(=O)c1ccccc1C2=O</smiles>

Rubiadin<smiles>COc1c(C)c(O)cc2c1C(=O)c1ccccc1C2=O</smiles>

Rubiadin 1-methylether<smiles>O=C1c2cccc(O)c2C(=O)c2c(O)cccc21</smiles>

1,8-Dihydroxy-anthraquinone<smiles>O=C(O)C1=CO[C@@H](O[C@@H]2O[C@H](CO)[C@@H](O)[C@H](O)[C@H]2O)C2C1C=C[C@]2(O)CO</smiles>

Monotropein<smiles>COC(=O)C1=CO[C@@H](O[C@@H]2O[C@H](CO)[C@@H](O)[C@H](O)[C@H]2O)C2C(CO)=CCC12</smiles>

Geniposide

Figure 1: Structure of standard and internal compounds.

TABLE 1: The MS/MS transitions and energy parameters of analytes and IS.

\begin{tabular}{lcccc}
\hline Compound & {$[\mathrm{M}-\mathrm{H}]^{-}(\mathrm{m} / z)$} & Quantitative ion $(\mathrm{m} / z)$ & Cone voltage $(\mathrm{V})$ & Collision energy $(\mathrm{eV})$ \\
\hline Rubiadin & 253.05 & 225.06 & 58 & 40 \\
Rubiadin 1-methyl ether & 267.06 & 252.04 & 50 & 18 \\
Monotropein & 389.11 & 191.03 & 40 & 15 \\
1,8-Dihydroxyanthraquinone & 239.04 & 211.04 & 30 & 35 \\
Geniposide & 387.13 & 225.07 & 36 & 8 \\
\hline
\end{tabular}

MO: steam the clean Morindae officinalis Radix for $0.5 \mathrm{~h}$, remove the woody cores while hot, cut into sections, and dry.

SMO: steam the clean Morindae officinalis Radix with salt water for $4.0 \mathrm{~h}$, until it is steamed thoroughly, remove the woody cores while hot, cut into sections and dry.

Then, MO and SMO were refluxed with $80 \%$ ethanol three times for $1 \mathrm{~h}$ each to make a combined filtrate. Then, the extracts were concentrated to $4 \mathrm{~g} \cdot \mathrm{mL}^{-1}$.

The contents of the three constituents in the MO and SMO extracts were detected by the same UPLC-MS/MS method, including the same column, mobile phase system, and column temperature as used for the pharmacokinetics and tissue distribution study. The contents of monotropein, rubiadin, and rubiadin 1-methyl ether in the MO and SMO extracts were $6.44,0.088$, and 0.174 and 3.95, 0.091, and $0.178 \mathrm{mg} / \mathrm{g}$, respectively. The concentrated extract was redissolved in distilled water.

2.6. Preparation of Biological Samples. $10 \mu \mathrm{L}$ of IS working solution and $400 \mu \mathrm{L}$ of methanol were added to $100 \mu \mathrm{L}$ plasma. The solution was vortexed for $10 \mathrm{~min}$ and then centrifuged at $12,000 \mathrm{rpm}$ for $5 \mathrm{~min}$. The supernatant was shifted to another centrifugation tube and dried with nitrogen at $37^{\circ} \mathrm{C}$. The residue was redissolved in $100 \mu \mathrm{L}$ acetonitrile and centrifuged for $5 \mathrm{~min}$ at $12,000 \mathrm{rpm}$. Five microliters of supernatant was drawn and analyzed by UPLC-MS/MS.
The whole tissue was cut into pieces and homogenized with ice-cold $0.9 \%(\mathrm{~m} / \mathrm{v})$ sodium chloride solution $(1: 9, \mathrm{w} / \mathrm{v})$. Using $50 \mu \mathrm{L}$ of tissue homogenate, the tissue samples were prepared in the same way as the plasma samples.

\subsection{Method Validation}

2.7.1. Specificity. Six individual sources of blank plasma were used to measure the specificity of this analysis method. Meanwhile, we also analyzed the lower limit of quantification (LLOQ) of samples, the IS in blank plasma samples, and plasma samples within $2 \mathrm{~h}$ of being subjected to the $\mathrm{MO}$ extract $(40 \mathrm{~g} / \mathrm{kg})$.

2.7.2. Calibration Curve and Sensitivity. The peak-area ratio of the compounds to the IS was plotted to obtain the linearity. The weighted least-squares linear regression (weighting factor $1 / X^{2}$ ) was applied to determine the regression equations. The lowest concentration in the calibration curve was determined as the LLOQ with signal-tonoise of $\geq 10$.

2.7.3. Precision, Accuracy, and Stability. Through evaluating three levels of QC samples $(n=5)$ after one day and then for three days in a row, we obtained the intra- and interday precision, along with the accuracy data. The permitted range was within $15 \%$. 


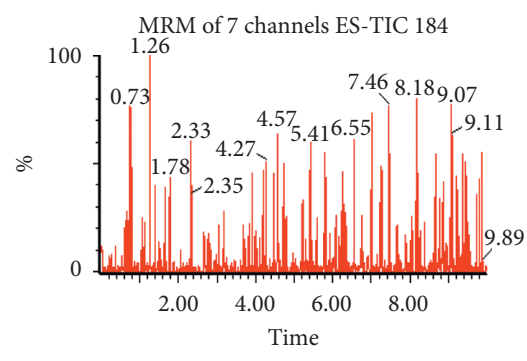

A

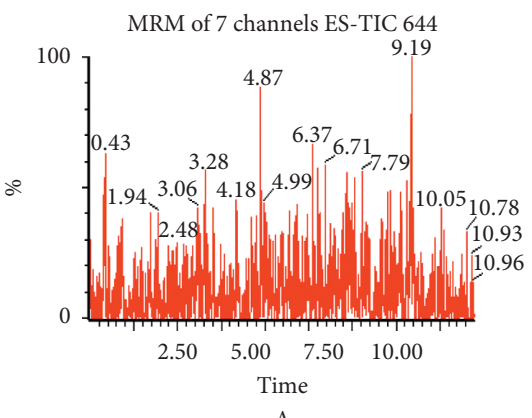

A

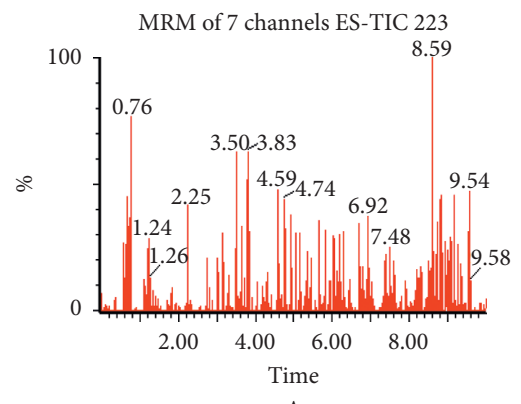

A

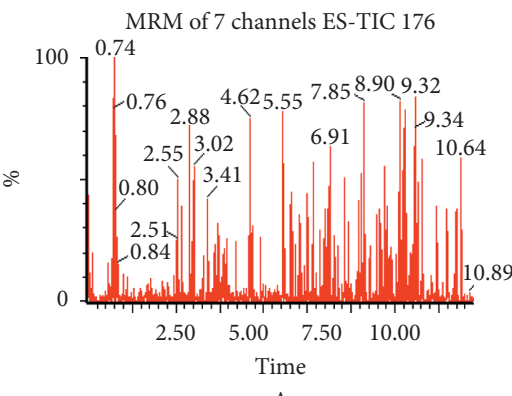

A

(a)
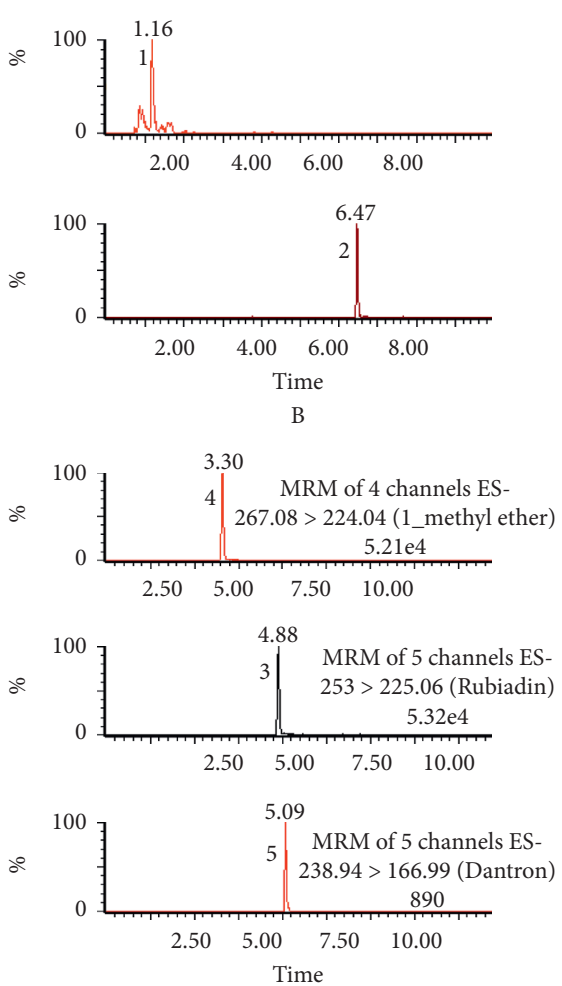

B
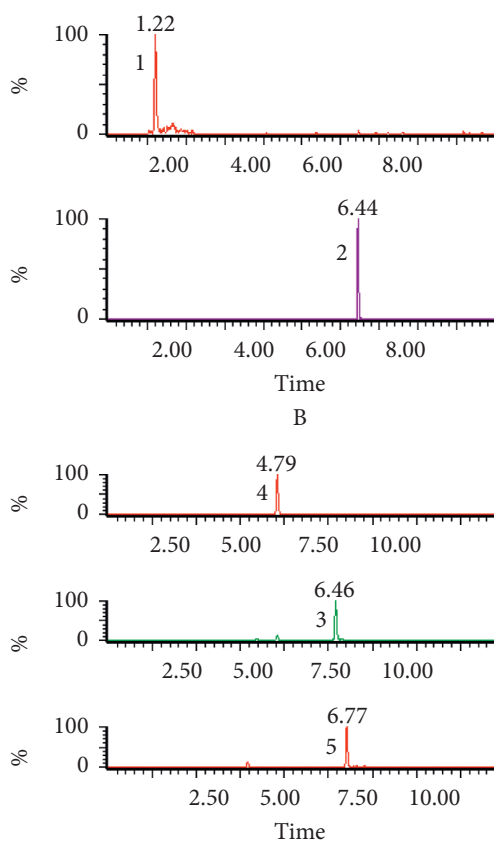

B

(b)
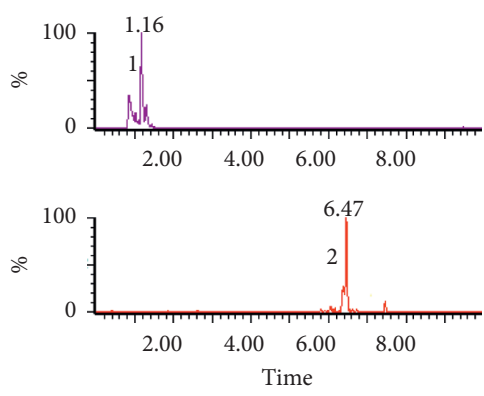

C
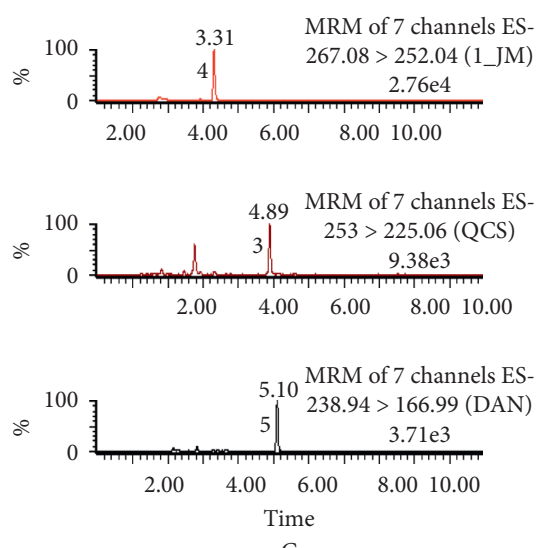

C
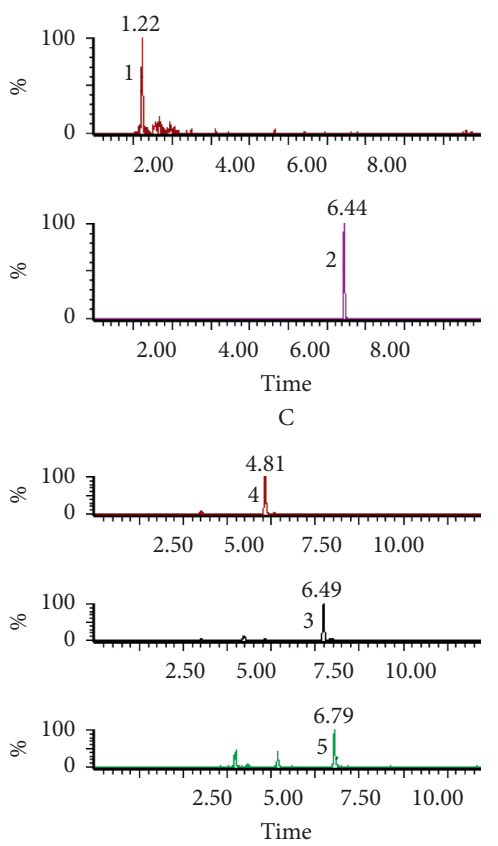

C

(c)

FIGURE 2: Multiple reaction monitoring (MRM) chromatograms of monotropein, rubiadin, rubiadin 1-methyl ether, and the IS in rat plasma: (a) blank plasma samples, (b) blank plasma samples spiked with three analytes at the lower limit of quantification (LLOQ), and (c) plasma samples at $2 \mathrm{~h}$ after the oral administration of $M$. officinalis extract (40 g/kg). (1) Monotropein, (2) geniposide, (3) rubiadin, (4) rubiadin 1-methyl ether, and (5) 1,8-dihydroxyanthraquinone. 
TABLE 2: Calibration curves and LLOQs of components in rat plasma and tissues.

\begin{tabular}{|c|c|c|c|c|c|}
\hline & Components & Calibration curves & $r^{2}$ & Range $(\mu \mathrm{g} / \mathrm{mL})$ & LLOQ $(\mu \mathrm{g} / \mathrm{mL})$ \\
\hline Plasma & $\begin{array}{c}\text { Rubiadin } \\
\text { Rubiadin 1-methyl ether } \\
\text { Monotropein }\end{array}$ & $\begin{array}{c}Y=3.32809 X+0.40361 \\
Y=1.08619 X+1.03779 \\
Y=0.0256 X-0.0026\end{array}$ & $\begin{array}{l}r^{2}=0.9936 \\
r^{2}=0.9919 \\
r^{2}=0.9943\end{array}$ & $\begin{array}{c}1.2 \sim 950 \\
1.3 \sim 1020 \\
0.047 \sim 37.5 \\
\end{array}$ & $\begin{array}{c}1.2 \\
1.3 \\
0.047 \\
\end{array}$ \\
\hline Liver & $\begin{array}{c}\text { Rubiadin } \\
\text { Rubiadin 1-methyl ether } \\
\text { Monotropein }\end{array}$ & $\begin{array}{c}Y=4.4365 X+0.629 \\
Y=5.0622 X+0.7667 \\
Y=0.0315 X-0.0741\end{array}$ & $\begin{array}{l}r^{2}=0.9928 \\
r^{2}=0.9911 \\
r^{2}=0.9915\end{array}$ & $\begin{array}{l}0.07 \sim 6.18 \\
0.05 \sim 4.66 \\
0.47 \sim 37.5 \\
\end{array}$ & $\begin{array}{l}0.07 \\
0.05 \\
0.47 \\
\end{array}$ \\
\hline Small intestine & $\begin{array}{c}\text { Rubiadin } \\
\text { Rubiadin 1-methyl ether } \\
\text { Monotropein }\end{array}$ & $\begin{array}{l}Y=3.4909 X-2.2398 \\
Y=6.4061 X-0.1149 \\
Y=0.0214 X-0.0251\end{array}$ & $\begin{array}{l}r^{2}=0.9907 \\
r^{2}=0.9926 \\
r^{2}=0.9969\end{array}$ & $\begin{array}{c}0.07 \sim 12.375 \\
0.092 \sim 9.2 \\
0.47 \sim 37.5 \\
\end{array}$ & $\begin{array}{c}0.07 \\
0.092 \\
0.47 \\
\end{array}$ \\
\hline Kidney & $\begin{array}{c}\text { Rubiadin } \\
\text { Rubiadin 1-methyl ether } \\
\text { Monotropein }\end{array}$ & $\begin{array}{l}Y=3.5819 X-0.9479 \\
Y=5.3299 X-0.6431 \\
Y=0.0333 X-0.0156\end{array}$ & $\begin{array}{l}r^{2}=0.9938 \\
r^{2}=0.9968 \\
r^{2}=0.9997\end{array}$ & $\begin{array}{l}0.07 \sim 6.18 \\
0.05 \sim 4.66 \\
0.47 \sim 37.5 \\
\end{array}$ & $\begin{array}{l}0.07 \\
0.05 \\
0.47\end{array}$ \\
\hline Lung & $\begin{array}{c}\text { Rubiadin } \\
\text { Rubiadin 1-methyl ether }\end{array}$ & $\begin{array}{l}Y=4.4389 X-0.7058 \\
Y=8.7095 X-2.3863\end{array}$ & $\begin{array}{l}r^{2}=0.9966 \\
r^{2}=0.9919\end{array}$ & $\begin{array}{l}0.07 \sim 6.18 \\
0.05 \sim 4.66\end{array}$ & $\begin{array}{l}0.07 \\
0.05\end{array}$ \\
\hline
\end{tabular}

TABle 3: Precision and accuracy for monotropein, rubiadin, and rubiadin 1-methyl ether in biological samples.

\begin{tabular}{|c|c|c|c|c|c|c|}
\hline \multirow{2}{*}{ Matrix } & \multirow{2}{*}{ Compounds } & \multirow{2}{*}{ Conc. $(\mu \mathrm{g} / \mathrm{mL})$} & \multicolumn{2}{|c|}{ Intra-day $(n=5)$} & \multicolumn{2}{|c|}{ Inter-day $(n=5)$} \\
\hline & & & Precision (RSD\%) & Accuracy (RE\%) & Precision (RSD\%) & Accuracy (RE\%) \\
\hline \multirow{9}{*}{ Plasma } & \multirow{6}{*}{ Rubiadin 1-methyl ether } & 3.6 & 1.23 & -8.06 & 0.21 & -3.06 \\
\hline & & 50.6 & 2.77 & -2.92 & 2.85 & -2.75 \\
\hline & & 712.5 & 5.96 & -2.01 & 2.79 & -4.15 \\
\hline & & 3.9 & 4.91 & -7.44 & 3.42 & -2.82 \\
\hline & & 54.6 & 2.33 & -0.33 & 1.73 & -2.44 \\
\hline & & 765 & 2.28 & -5.45 & 3.33 & -3.53 \\
\hline & \multirow{3}{*}{ Monotropein } & 0.14 & 3.51 & -1.42 & 3.27 & -2.13 \\
\hline & & 2 & 1.25 & -3.4 & 1.93 & -0.9 \\
\hline & & 28.1 & 2.79 & -3.36 & 0.76 & -2.9 \\
\hline \multirow{9}{*}{ Small intestine } & \multirow{6}{*}{ Rubiadin 1-methyl ether } & 0.21 & 4.44 & -1.43 & 4.75 & -2.86 \\
\hline & & 0.98 & 4.43 & -0.1 & 4.62 & 1.12 \\
\hline & & 4.63 & 4.55 & -0.41 & 1.15 & -0.82 \\
\hline & & 0.15 & 1.66 & -0.67 & 1.12 & -2.67 \\
\hline & & 0.72 & 5.83 & -0.69 & 5.05 & 0.14 \\
\hline & & 3.49 & 5.55 & -0.14 & 2.85 & 0.06 \\
\hline & \multirow{3}{*}{ Monotropein } & 1.41 & 5.55 & -0.07 & 1.72 & -0.71 \\
\hline & & 6.2 & 1.63 & -1.29 & 2.48 & 0.16 \\
\hline & & 28.13 & 3.49 & -0.64 & 3.41 & -0.25 \\
\hline \multirow{9}{*}{ Liver } & \multirow{6}{*}{ Rubiadin 1-methyl ether } & 0.21 & 3.43 & -0.48 & 1.9 & -0.48 \\
\hline & & 0.98 & 1.3 & -0.51 & 2.89 & -0.41 \\
\hline & & 4.63 & 2.5 & -0.13 & 3.33 & 0.04 \\
\hline & & 0.15 & 2.42 & -4 & 4.62 & 1.33 \\
\hline & & 0.72 & 1.57 & -0.28 & 5.56 & -1.25 \\
\hline & & 3.49 & 2.27 & -0.11 & 2.54 & -0.2 \\
\hline & \multirow{3}{*}{ Monotropein } & 1.41 & 4.36 & -0.35 & 4.75 & -0.78 \\
\hline & & 6.2 & 1.32 & -0.81 & 2.2 & 0.48 \\
\hline & & 28.13 & 5.95 & -2.03 & 3.82 & 0.03 \\
\hline \multirow{9}{*}{ Kidney } & \multirow{6}{*}{ Rubiadin 1-methyl ether } & 0.21 & 1.86 & -0.05 & 5.96 & -3.81 \\
\hline & & 0.98 & 1.56 & -0.51 & 3.71 & -0.51 \\
\hline & & 4.63 & 3.23 & -0.11 & 2.36 & -0.13 \\
\hline & & 0.15 & 3.33 & -2 & 4.18 & -2.67 \\
\hline & & 0.72 & 3.06 & -0.56 & 4.35 & -0.28 \\
\hline & & 3.49 & 2.17 & -0.14 & 4.35 & 0.09 \\
\hline & \multirow{3}{*}{ Monotropein } & 1.41 & 1.5 & -0.78 & 2.54 & -0.64 \\
\hline & & 6.2 & 3.74 & -1.29 & 3.5 & -0.81 \\
\hline & & 28.13 & 1.21 & 0.02 & 3.63 & -0.5 \\
\hline
\end{tabular}


TABle 3: Continued.

\begin{tabular}{|c|c|c|c|c|c|c|}
\hline \multirow{2}{*}{ Matrix } & \multirow{2}{*}{ Compounds } & \multirow{2}{*}{ Conc. $(\mu \mathrm{g} / \mathrm{mL})$} & \multicolumn{2}{|c|}{ Intra-day $(n=5)$} & \multicolumn{2}{|c|}{ Inter-day $(n=5)$} \\
\hline & & & Precision (RSD\%) & Accuracy (RE\%) & Precision (RSD\%) & Accuracy (RE\%) \\
\hline \multirow{6}{*}{ Lung } & & 0.21 & 1.2 & -0.38 & 2.15 & 0.48 \\
\hline & Rubiadin & 0.98 & 2.88 & -0.51 & 1.8 & 0.2 \\
\hline & & 4.63 & 5.93 & -0.04 & 3.33 & -0.17 \\
\hline & & 0.15 & 5.04 & -2.67 & 0.6 & -1.33 \\
\hline & Rubiadin 1-methyl ether & 0.72 & 1.33 & -0.28 & 1.59 & 0.28 \\
\hline & & 3.49 & 5.52 & -0.06 & 1.72 & 0.06 \\
\hline
\end{tabular}

TABLE 4: Stability of the three components in rat plasma and tissues $(n=5)$.

\begin{tabular}{|c|c|c|c|c|c|c|c|c|c|c|}
\hline \multirow{3}{*}{ Matrix } & \multirow{3}{*}{ Compounds } & \multirow{3}{*}{ Conc $(\mu \mathrm{g} / \mathrm{mL})$} & \multicolumn{2}{|c|}{ At $25^{\circ} \mathrm{C}$ for $12 \mathrm{~h}$} & \multicolumn{2}{|c|}{$\begin{array}{c}-20^{\circ} \mathrm{C} \text { for } 30 \\
\text { days }\end{array}$} & \multicolumn{2}{|c|}{$\begin{array}{l}\text { Autosampler } \\
\text { at } 4^{\circ} \mathrm{C} \text { for } 24 \mathrm{~h}\end{array}$} & \multicolumn{2}{|c|}{$\begin{array}{c}\text { Freeze-thaw } \\
\text { cycles }\end{array}$} \\
\hline & & & \multirow{2}{*}{\multicolumn{2}{|c|}{$\mathrm{RSD} \%$}} & \multirow{2}{*}{\multicolumn{2}{|c|}{$\begin{array}{l}\mathrm{RSD} \\
\%\end{array}$}} & \multirow{2}{*}{\multicolumn{2}{|c|}{$\begin{array}{lc} & \text { RSD } \\
\text { RE\% } & \%\end{array}$}} & \multirow{2}{*}{\multicolumn{2}{|c|}{$\begin{array}{c}\text { RSD } \\
\% \\
\end{array}$}} \\
\hline & & & & & & & & & & \\
\hline \multirow{9}{*}{ Plasma } & \multirow{6}{*}{ Rubiadin 1-methyl ether } & 3.6 & -6.23 & 4.18 & -3.16 & 2.13 & -1.72 & 3.13 & -0.92 & 4.22 \\
\hline & & 50.6 & -1.62 & 3.86 & -2.38 & 1.45 & -0.97 & 4.13 & -1.35 & 1.45 \\
\hline & & 712.5 & -4.1 & 4.41 & -2.46 & 3.81 & -1.76 & 3.56 & -2.34 & 6.27 \\
\hline & & 3.9 & -1.79 & 1.63 & -3.26 & 2.36 & -1.49 & 2.48 & -0.94 & 3.85 \\
\hline & & 54.6 & -4.21 & 3.41 & -0.23 & 3.42 & -1.65 & 3.53 & -1.35 & 3.18 \\
\hline & & 765 & -5.1 & 2.81 & -0.86 & 3.13 & -3.14 & 1.73 & -3.71 & 2.45 \\
\hline & \multirow{3}{*}{ Monotropein } & 0.14 & -0.14 & 3.21 & -1.21 & 2.51 & -2.39 & 2.46 & -1.95 & 2.99 \\
\hline & & 2 & -0.13 & 2.41 & -0.24 & 2.91 & -0.46 & 3.74 & -0.52 & 4.82 \\
\hline & & 28.1 & -0.21 & 3.92 & -1.36 & 4.82 & -1.23 & 3.69 & -2.89 & 3.91 \\
\hline \multirow{9}{*}{ Small intestine } & \multirow{7}{*}{ Rubiadin 1-methyl ether } & 0.21 & -0.07 & 2.59 & -0.35 & 1.98 & -0.64 & 5.7 & -0.45 & 3.54 \\
\hline & & 0.98 & -0.57 & 2.96 & -2.71 & 1.14 & -1.65 & 5.79 & -0.38 & 3.85 \\
\hline & & 4.63 & -1.47 & 5.43 & -0.87 & 8.51 & -1.67 & 2.97 & 0.39 & 2.68 \\
\hline & & 0.15 & -0.69 & 7.53 & -1.25 & 0.65 & -0.42 & 2.74 & -6.57 & 8.41 \\
\hline & & 0.72 & -0.85 & 6.41 & -0.25 & 4.23 & -0.62 & 2.19 & -0.57 & 1.88 \\
\hline & & 3.49 & 0.25 & 2.47 & 1.35 & 2.49 & 0.48 & 7.29 & -0.74 & 8.82 \\
\hline & & 1.41 & -0.63 & 3.61 & -0.85 & 1.58 & -0.35 & 4.86 & -0.99 & 1.25 \\
\hline & \multirow[t]{2}{*}{ Monotropein } & 6.2 & -1.41 & 5.93 & -0.71 & 3.87 & -0.66 & 5.87 & 0.15 & 6.02 \\
\hline & & 28.13 & -2.27 & 4.86 & -0.12 & 4.64 & -0.01 & 6.84 & -0.01 & 2.57 \\
\hline \multirow{9}{*}{ Liver } & \multirow{6}{*}{ Rubiadin 1-methyl ether } & 0.21 & -0.52 & 1.38 & -0.54 & 1.28 & 0.26 & 1.07 & -5.57 & 6.37 \\
\hline & & 0.98 & -2.43 & 0.86 & -0.8 & 8.16 & -0.33 & 2.08 & 0.25 & 4.23 \\
\hline & & 4.63 & -0.57 & 2.36 & 1.57 & 5.37 & -2.36 & 2.97 & 0.27 & 3.67 \\
\hline & & 0.15 & -2.1 & 5.64 & -0.58 & 3.76 & 0.54 & 2.97 & -1.52 & 5.24 \\
\hline & & 0.72 & -3.42 & 6.84 & -0.45 & 1.53 & -3.81 & 7.23 & -5.42 & 4.27 \\
\hline & & 3.49 & 0.42 & 2.23 & -2.58 & 2.45 & -0.66 & 3.88 & -0.68 & 2.68 \\
\hline & \multirow{3}{*}{ Monotropein } & 1.41 & -0.85 & 0.41 & -1.84 & 6.21 & -2.98 & 1.78 & -1.77 & 3.72 \\
\hline & & 6.2 & 0.56 & 1.68 & -0.52 & 4.2 & 0.23 & 2.87 & -0.84 & 2.98 \\
\hline & & 28.13 & -1.71 & 3.69 & -0.65 & 3.51 & -3.16 & 2.97 & 0.09 & 1.96 \\
\hline \multirow{9}{*}{ Kidney } & & 0.21 & -0.42 & 0.32 & -0.54 & 6.45 & -0.45 & 7.95 & -0.57 & 1.87 \\
\hline & Rubiadin & 0.98 & -0.38 & 0.98 & -2.45 & 2.76 & -6.72 & 4.56 & -1.57 & 6.41 \\
\hline & & 4.63 & -0.17 & 3.76 & -0.67 & 0.64 & 1.46 & 2.17 & 0.38 & 5.13 \\
\hline & & 0.15 & 0.35 & 2.94 & -5.1 & 2.76 & -0.36 & 1.56 & 0.15 & 2.51 \\
\hline & Rubiadin 1-methyl ether & 0.72 & 0.24 & 1.9 & -2.49 & 3.57 & -0.68 & 2.35 & -0.54 & 5.42 \\
\hline & & 3.49 & -3.58 & 4.35 & 0.54 & 1.94 & -0.36 & 3.56 & -1.57 & 4.23 \\
\hline & & 1.41 & -0.28 & 6.72 & -0.86 & 1.62 & -0.18 & 3.5 & 0.24 & 3.69 \\
\hline & Monotropein & 6.2 & -0.67 & 6.42 & -0.46 & 7.56 & -0.57 & 5.44 & 0.07 & 4.29 \\
\hline & & 28.13 & -0.68 & 0.34 & -0.27 & 6.52 & 0.25 & 2.34 & -0.57 & 2.86 \\
\hline & & 0.21 & 0.65 & 0.86 & 0.42 & 3.87 & -0.24 & 5.44 & -0.38 & 5.71 \\
\hline & Rubiadin & 0.98 & 0.38 & 3.57 & 0.48 & 0.68 & -0.54 & 3.46 & -1.57 & 5.74 \\
\hline & & 4.63 & 0.68 & 6.84 & -5.71 & 0.42 & -0.57 & 3.55 & 0.35 & 6.7 \\
\hline Lung & & 0.15 & -0.57 & 6.42 & -1.54 & 0.35 & -8.51 & 7.62 & -2.57 & 3.85 \\
\hline & Rubiadin 1-methyl ether & 0.72 & -5.71 & 2.43 & -0.55 & 7.36 & -0.65 & 3.57 & -4.85 & 4.42 \\
\hline & & 3.49 & -0.06 & 2.46 & 0.05 & 4.51 & -2.42 & 6.58 & -0.53 & 2.46 \\
\hline
\end{tabular}


TABle 5: Extraction recoveries in rat plasma and tissues $(n=5)$.

\begin{tabular}{|c|c|c|c|c|}
\hline Matrix & Compounds & Conc. $(\mu \mathrm{g} / \mathrm{mL})$ & Recoveries (\%) & RSD (\%) \\
\hline \multirow{9}{*}{ Plasma } & \multirow{6}{*}{ Rubiadin 1-methyl ether } & 3.6 & 88.34 & 7.03 \\
\hline & & 50.6 & 95.08 & 1.89 \\
\hline & & 712.5 & 96.39 & 0.93 \\
\hline & & 3.9 & 81.89 & 3.24 \\
\hline & & 54.6 & 93.42 & 3.13 \\
\hline & & 765 & 94.05 & 1.44 \\
\hline & \multirow{3}{*}{ Monotropein } & 0.14 & 95.51 & 3.66 \\
\hline & & 2 & 91.97 & 3.88 \\
\hline & & 28.1 & 97.60 & 1.10 \\
\hline \multirow{9}{*}{ Small intestine } & \multirow{3}{*}{ Rubiadin } & 0.21 & 85.24 & 3.91 \\
\hline & & 0.98 & 91.09 & 4.05 \\
\hline & & 4.63 & 96.21 & 1.15 \\
\hline & \multirow{3}{*}{ Rubiadin 1-methyl ether } & 0.15 & 92.44 & 2.20 \\
\hline & & 0.72 & 91.39 & 8.03 \\
\hline & & 3.49 & 86.97 & 3.60 \\
\hline & \multirow{3}{*}{ Monotropein } & 1.41 & 98.27 & 1.83 \\
\hline & & 6.2 & 98.64 & 1.03 \\
\hline & & 28.13 & 92.95 & 0.02 \\
\hline \multirow{9}{*}{ Liver } & \multirow{3}{*}{ Rubiadin } & 0.21 & 89.37 & 2.15 \\
\hline & & 0.98 & 88.98 & 1.13 \\
\hline & & 4.63 & 94.16 & 2.78 \\
\hline & \multirow{3}{*}{ Rubiadin 1-methyl ether } & 0.15 & 88.89 & 7.21 \\
\hline & & 0.72 & 85.97 & 3.28 \\
\hline & & 3.49 & 90.84 & 1.87 \\
\hline & \multirow{3}{*}{ Monotropein } & 1.41 & 97.07 & 3.25 \\
\hline & & 6.2 & 97.67 & 1.61 \\
\hline & & 28.13 & 96.41 & 0.91 \\
\hline \multirow{9}{*}{ Kidney } & \multirow{3}{*}{ Rubiadin } & 0.21 & 88.73 & 4.56 \\
\hline & & 0.98 & 82.07 & 8.44 \\
\hline & & 4.63 & 93.59 & 4.07 \\
\hline & \multirow{3}{*}{ Rubiadin 1-methyl ether } & 0.15 & 88.89 & 4.26 \\
\hline & & 0.72 & 93.61 & 6.61 \\
\hline & & 3.49 & 89.98 & 3.55 \\
\hline & \multirow{3}{*}{ Monotropein } & 1.41 & 93.95 & 4.96 \\
\hline & & 6.2 & 93.41 & 4.95 \\
\hline & & 28.13 & 94.47 & 0.87 \\
\hline \multirow{6}{*}{ Lung } & \multirow{3}{*}{ Rubiadin } & 0.21 & 80.63 & 2.46 \\
\hline & & 0.98 & 80.07 & 6.59 \\
\hline & & 4.63 & 83.97 & 1.47 \\
\hline & \multirow{3}{*}{ Rubiadin 1-methyl ether } & 0.15 & 91.11 & 3.30 \\
\hline & & 0.72 & 86.67 & 5.77 \\
\hline & & 3.49 & 88.96 & 5.08 \\
\hline
\end{tabular}

The QC samples used for the recovery QC samples were used to judge the stability, and the storage conditions were as follows: (1) freeze-thaw stability, (2) at room temperature for $4 \mathrm{~h}$, and (3) put into the autosampler for $8 \mathrm{~h}$, and (4) at $-20^{\circ} \mathrm{C}$ for 30 days. The permitted range was within $15 \%$.

2.7.4. Extraction Recovery and Matrix Effect. Three different QC sample concentrations were used for the recovery experiment. The absolute extraction recovery was measured as the ratio of the QC sample concentration extracted from the blank plasma/tissue to those in the QC samples.

The peak areas of the biosamples extracted in blank plasma/tissue versus those dissolved in methanol solution were measured to obtain the matrix effect.
2.8. Analysis of Pharmacokinetics. The SD rats $(6$, male) were housed at $22 \pm 2^{\circ} \mathrm{C}$ and fasted for $12 \mathrm{~h}$ with drinking water available ad libitum. MO and SMO ethanol extract were orally administered at a dose of $40 \mathrm{~g} / \mathrm{kg}$ (equivalent to 6.44 and $3.95 \mathrm{mg} / \mathrm{kg}$ of monotropein, 0.088 and $0.091 \mathrm{mg} / \mathrm{kg}$ of rubiadin, and 0.174 and $0.178 \mathrm{mg} / \mathrm{kg}$ of rubiadin 1 -methyl ether). Blood samples $(0.5 \mathrm{~mL})$ were collected from the venous plexus of the eye socket at $0.083,0.25,0.5,1.0,1.5$, 2.0, 2.5, 4.0, 6.0, 12.0, and $24 \mathrm{~h}$ under anesthesia. Then, the blood samples were centrifuged at $4500 \mathrm{rpm}$ for $10 \mathrm{~min}$, and then the plasma was immediately transferred to new tubes and stored at $-80^{\circ} \mathrm{C}$ until analysis. The pharmacokinetic parameters were calculated using DAS software (3.0 version, China Food and Drug Administration) based on noncompartmental method. All data were recorded as mean \pm SD. 


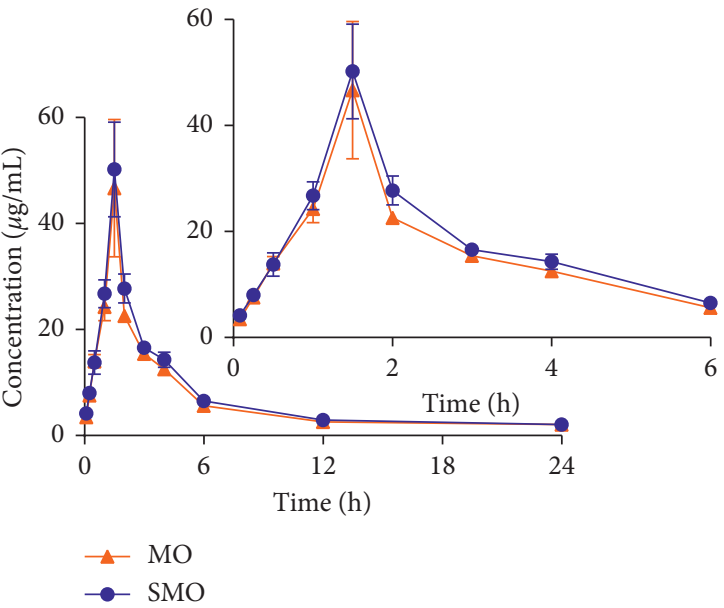

(a)

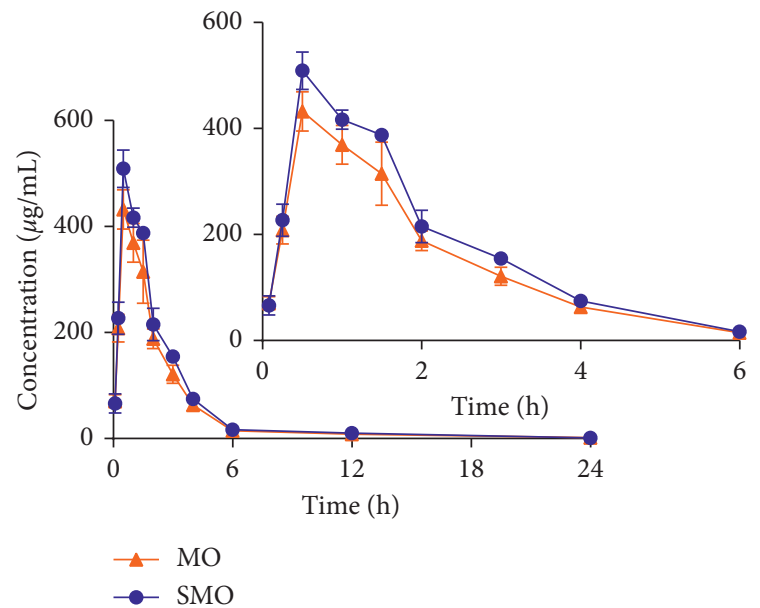

(b)

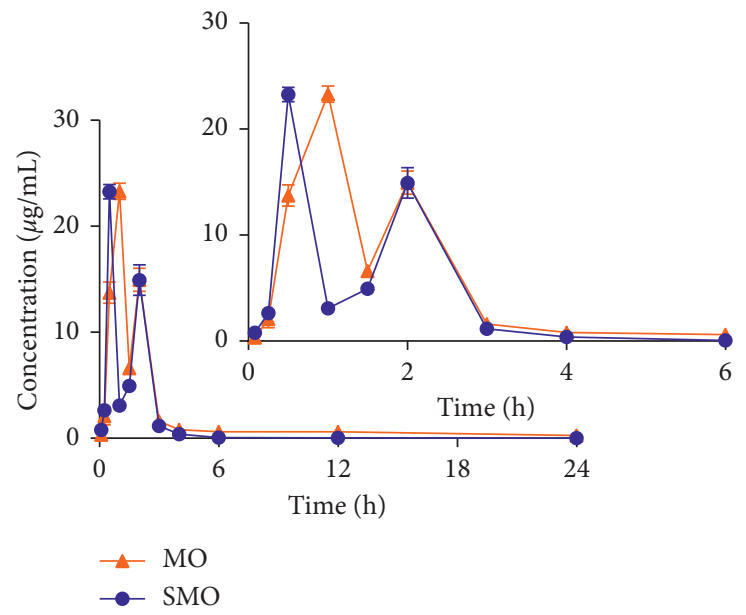

(c)

FIGURE 3: Mean $( \pm S D, n=6)$ plasma concentrations of rubiadin, rubiadin 1-methyl ether, and monotropein after oral administration of $M$. officinalis without wood (MO) and salt-processed product (SMO) extracts (40 g/kg). (a) Rubiadin, (b) rubiadin 1-methyl ether, and (c) monotropein.

TABle 6: Pharmacokinetic parameters of the three components in rat plasma $(n=6)$.

\begin{tabular}{lccccccc}
\hline \multirow{2}{*}{ Parameters } & \multirow{2}{*}{ Units } & \multicolumn{2}{c}{ Mean (rubiadin) } & \multicolumn{2}{c}{ Mean (rubiadin 1-methyl ether) } & \multicolumn{2}{c}{ Mean (monotropein) } \\
& & MO & SMO & MO & SMO & MO & SMO \\
\hline AUC $(0-t)$ & $\mu \mathrm{g} / \mathrm{L} * \mathrm{~h}$ & $152.252 \pm 7.098$ & $169.355 \pm 8.836$ & $1059.49 \pm 98.081$ & $1244.329 \pm 82.805$ & $44.22 \pm 0.932$ & $26.941 \pm 1.423$ \\
AUC $(0-\infty)$ & $\mu \mathrm{g} / \mathrm{L} * \mathrm{~h}$ & $153.816 \pm 7.169$ & $176.441 \pm 18.283$ & $1074.351 \pm 102.019$ & $1253.526 \pm 84.691$ & $48.952 \pm 1.559$ & $27.045 \pm 1.338$ \\
MRT $(0-t)$ & $\mathrm{H}$ & $6.062 \pm 0.272$ & $5.89 \pm 0.265$ & $2.908 \pm 0.151$ & $2.855 \pm 0.15$ & $3.863 \pm 0.184$ & $1.686 \pm 0.025$ \\
$t 1 / 2 z$ & $\mathrm{H}$ & $3.838 \pm 0.196$ & $5.006 \pm 3.383$ & $5.379 \pm 1.514$ & $4.27 \pm 0.865$ & $12.291 \pm 2.09$ & $5.611 \pm 3.958$ \\
$T_{\max }$ & $\mathrm{H}$ & $1.5 \pm 0.00$ & $1.5 \pm 0.00$ & $0.5 \pm 0.00$ & $0.5 \pm 0.00$ & $1.0 \pm 0.00$ & $0.5 \pm 0.00$ \\
$C_{\max }$ & $\mu \mathrm{g} / \mathrm{L}$ & $46.667 \pm 12.941$ & $50.2 \pm 8.903$ & $432.367 \pm 37.012$ & $509 \pm 35.133$ & $23.298 \pm 0.764$ & $23.253 \pm 0.676$ \\
$V / F$ & $\mathrm{~L} / \mathrm{g}$ & $1.442 \pm 0.090$ & $1.579 \pm 0.842$ & $0.287 \pm 0.067$ & $0.196 \pm 0.363$ & $14.447 \pm 2.143$ & $12.239 \pm 9.027$ \\
$C L / F$ & $\mathrm{~L} / \mathrm{g} \cdot \mathrm{h}$ & $0.261 \pm 0.012$ & $0.228 \pm 0.022$ & $0.037 \pm 0.372$ & $0.032 \pm 0.210$ & $0.818 \pm 0.265$ & $1.482 \pm 0.750$ \\
\hline
\end{tabular}




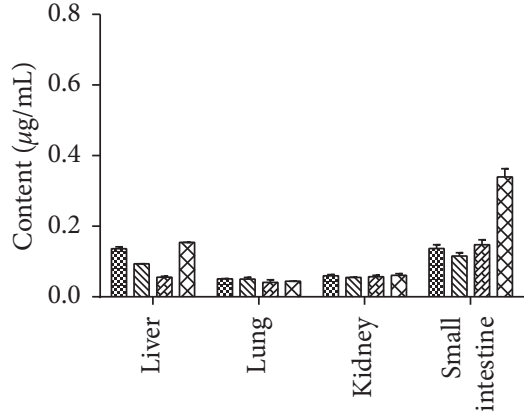

Tissues

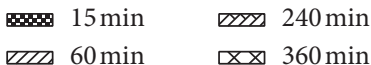

एב] $60 \mathrm{~min} \quad$ एख $360 \mathrm{~min}$

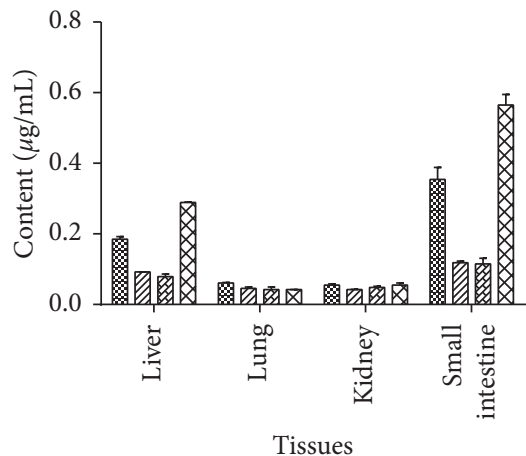

\$2 $15 \mathrm{~min}$

DI7 $60 \mathrm{~min}$

Eyz $240 \mathrm{~min}$

एX $360 \mathrm{~min}$

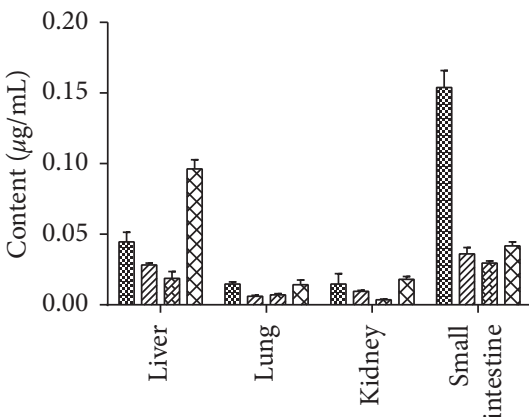

Tissues

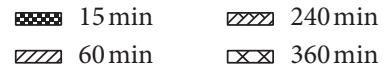

(a)

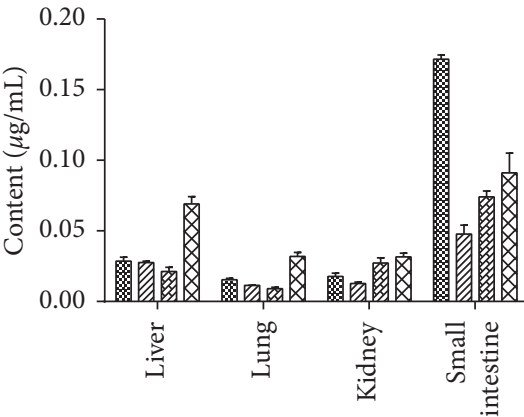

Tissues

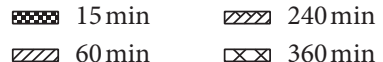

(b)

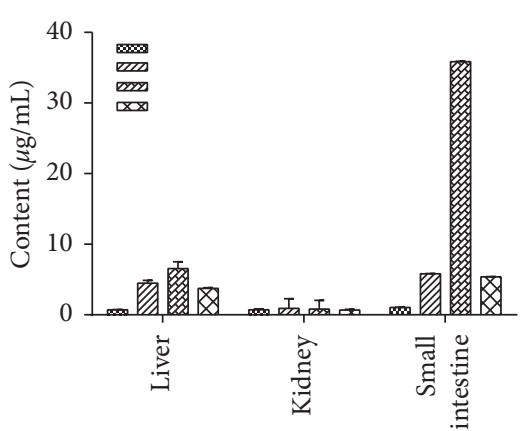

Tissues
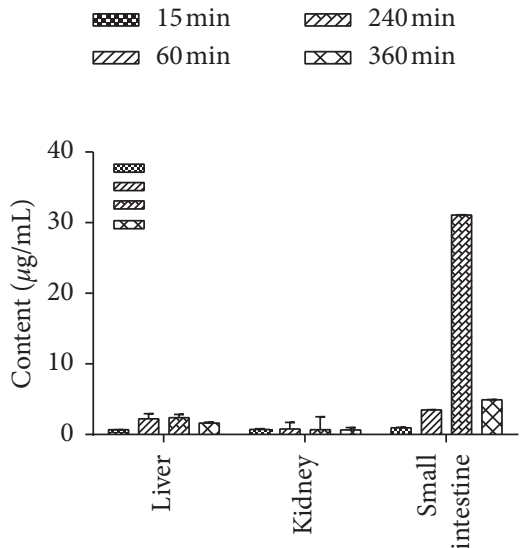

Tissues

$\$ 15 \mathrm{~min}$ सy $240 \mathrm{~min}$ DIT $60 \mathrm{~min} \quad$ एX $360 \mathrm{~min}$

Figure 4: Concentrations of rubiadin-1-methyl ether, rubiadin, and monotropein in rat tissues at $15 \mathrm{~min}, 60 \mathrm{~min}, 240 \mathrm{~min}$, and $360 \mathrm{~min}$ after intragastric administration of MO and SMO extracts (mean \pm SD, $n=6$ ). (a) Tissue distribution of the MO extract and (b) tissue distribution of the SMO extract. (1) Rubiadin-1-methyl ether, (2) rubiadin, and (3) monotropein.

2.9. Analysis of Tissue Distribution. MO and SMO ethanol extract $(40 \mathrm{~g} / \mathrm{kg})$ was orally administered to SD rats (weight, $180-220 \mathrm{~g}, n=6)$, at $0.25,1,3$, and $6 \mathrm{~h}$, respectively. Heart, liver, spleen, stomach, kidney, brain, and small intestine tissues were extracted, washed with saline, blotted with filter paper, weighed, and then stored at $-80^{\circ} \mathrm{C}$.

\section{Results and Discussion}

3.1. Liquid Chromatography Optimization. We tested mobile phase and gradient elution programs to determine the best chromatographic performance. With the mobile phase of water (containing $0.1 \%$ formic acid) and acetonitrile (containing $0.1 \%$ formic acid), the responses of the three components and two ISs were considerably better. To reach a better and more rapid separation effect, we optimized the gradient elution program.

3.2. Mass Spectrometry Optimization. We carried out both positive and negative modes in mass spectrometry. Three components and the ISs all had high responses under the negative mode. The MS/MS transitions and parameters are given in Table 1.
3.3. Extraction Procedure Optimization. Protein precipitation (PP) was used for the pretreatment of biological samples. We applied methanol and acetonitrile for PP. The samples had better peak shapes and recovery after being treated by acetonitrile, so we chose acetonitrile for further $\mathrm{PP}$ in this study.

\subsection{Method Validation}

3.4.1. Specificity. The MRM chromatograms of three components and the ISs are shown in Figure 2. The peak separation was better for the three components and the ISs under the established UPLC-MS/MS conditions, with no significant interference and no cross interference.

3.4.2. Linearity and Sensitivity. The internal standard method was applied for the establishment of calibration curves, which showed good linearity $\left(r^{2}>0.9907\right)$ in the linear ranges. The regression equations, correlation coefficients, linear ranges, and LLOQs of the three components in the plasma and tissues are listed in Table 2. 
3.4.3. Precision, Accuracy, and Stability. The range of intra and interday precisions was $0.21 \%$ to $5.96 \%$, and the accuracy range was from $-8.06 \%$ to $1.33 \%$ for QC samples in plasma and tissues. These values are in the acceptable range, and the results are shown in Table 3 .

Table 4 shows the results for the stability of the plasma and tissue samples in different conditions. They are also in the acceptable range.

3.4.4. Extraction Recovery and Matrix Effect. The range of extraction recoveries was 80.07 98.64\% for the three QC sample levels (Table 5). These data indicate that the sample treatment method is reasonable. No significant effect of endogenous substances was identified.

3.5. Pharmacokinetics Study. After oral administration of $\mathrm{MO}$ and SMO extract $(40.0 \mathrm{~g} / \mathrm{kg})$, we successfully determined the concentrations of monotropein, rubiadin, and rubiadin 1methyl ether in rat plasma using the established method. Figure 3 shows the mean plasma concentration-time profiles. Table 6 displays the pharmacokinetic parameters.

The concentration of monotropein in SMO reached $C_{\max }$ in $0.5 \mathrm{~h}$, whereas the $T_{\max }$ of monotropein in the MO groups was $1.0 \mathrm{~h}$, which indicates that monotropein was quickly adsorbed into the blood after oral administration. The $T_{\max }$ for rubiadin was $1.5 \mathrm{~h}$, longer than that for rubiadin 1 methyl ether, whereas the $C_{\max }$ for rubiadin 1-methyl ether was the highest, especially in the SMO groups. In the plasma concentration-time curves of monotropein, an obvious double-peak phenomenon was found, which is related to enterohepatic recirculation. The pharmacokinetics properties shown in the present assay could be helpful for further studies on the pharmacokinetics of MO extract and further applications in different processing methods.

3.6. Tissue Distribution. The concentrations of monotropein, rubiadin, and rubiadin 1-methyl ether in the liver, kidney, lung, and small intestine were determined at $0.25,1,3$, and $6 \mathrm{~h}$ after administration. The results are shown in Figure 4. All compounds were detected in tissues, except for monotropein, which could not be quantified in the lung tissue. This result indicates that monotropein might be rapidly transformed into its metabolites in the lungs, or the content of monotropein in the lung was lower. Meanwhile, all three components had higher concentrations in the small intestine, especially rubiadin and rubiadin 1-methyl ether in the SMO groups.

\section{Conclusion}

In this study, we established an efficient and accurate UPLCMS/MS method for the determination of three components from MO and SMO in plasma and tissues after oral administration in rats. Meanwhile, this is the first simultaneous determination of monotropein, rubiadin, and rubiadin-1methyl ether in rat plasma and tissues. The study examined the pharmacokinetics and tissue distribution. The information found might partially illustrate their metabolic mechanisms in vivo, as well as provide a scientific basis for the strengthening of kidney-yang using the salt-processed principle of $M$. officinalis because of better bioavailability.

\section{Data Availability}

The data used to support the findings of this study are included within the article.

\section{Conflicts of Interest}

The authors declare no conflicts of interest.

\section{Acknowledgments}

This research was supported by the National Natural Science Foundation of China (Grant nos. 81473350 and 81874345).

\section{References}

[1] Chinese Pharmacopoeia Commission, Pharmacopoeia of People's Republic of China, China Medical Science and Technology Press, Beijjing, China, 2015.

[2] K. Jiang, D. Huang, D. Zhang et al., "Investigation of inulins from the roots of Morinda officinalis for potential therapeutic application as anti-osteoporosis agent," International Journal of Biological Macromolecules, vol. 120, no. A, pp. 170-179, 2018.

[3] Y. K. Lee, H. J. Bang, J. B. Oh, and W. K. Whang, "Bioassayguided isolated compounds from Morinda officinalis inhibit Alzheimer's disease pathologies," Molecules, vol. 22, no. 10, pp. 1637-1649, 2017.

[4] T. Xia, X. Dong, L. Lin et al., "Metabolomics profiling provides valuable insights into the underlying mechanisms of Morinda officinalis on protecting glucocorticoid-induced osteoporosis," Journal of Pharmaceutical and Biomedical Analysis, vol. 166, pp. 336-346, 2019.

[5] L. Z. Xu, D. F. Xu, Y. Han et al., "BDNF-GSK-3 $\beta-\beta$-Catenin pathway in the $\mathrm{mPFC}$ is involved in antidepressant-like effects of Morinda officinalis oligosaccharides in rats," The International Journal of Neuropsychopharmacology, vol. 20, no. 1, pp. 83-93, 2017.

[6] J.-H. Zhang, H.-L. Xin, Y.-M. Xu et al., "Morinda officinalis How-a comprehensive review of traditional uses, phytochemistry and pharmacology," Journal of Ethnopharmacology, vol. 213, pp. 230-255, 2018.

[7] C. Cui, M. Yang, Z. Yao et al., "Antidepressant active constituents in the roots of Morinda officinalis How," Zhongguo Zhong Yao Za Zhi, vol. 20, no. 1, pp. 62-63, 1995.

[8] S. Li, Q. Ouyang, X. Tan, S. Shi, and Z. Yao, "Chemical constituents of Morinda officinalis How," Zhongguo Zhong Yao Za Zhi, vol. 16, no. 11, pp. 675-676, 1991.

[9] M. Yoshikawa, S. Yamaguchi, H. Nishisaka, J. Yamahara, and N. Murakami, "Chemical constituents of chinese natural medicine, morindae radix, the dried roots of Morinda officinalis How: structures of morindolide and morofficinaloside," Chemical \& Pharmaceutical Bulletin, vol. 43, no. 9, pp. 1462-1465, 1995.

[10] Z. Z. Zhao, Z. T. Liang, K. Chan, G. H. Lu, E. L. M. Lee, and H. B. Chen, "A unique issue in the standardization of Chinese materia medica: processing," Planta Medica, vol. 76, 2010.

[11] B. C. Cai, K. M. Qin, H. Wu, H. Cai, T. L. Lu, and X. D. Zhang, "Chemical mechanism during Chinese medicine processing," 
Progress in Chemical and Biochemical Research, vol. 24, pp. 637-649, 2012.

[12] C. Deng, L. Han, Y. Q. Zhang, and Y. Jiang, "Changes of chemical constituents in eucommiae cortex before and after processed with salt," Chin Tradit Pat Med, vol. 37, pp. 2464-2468, 2015.

[13] Z. H. Wang, J. Fu, L. B. Wu, X. Jiang, L. F. Huang, and S. L. Chen, "Changes of chemical constituents in psoraleae fructus before and after salted based on UPLC/Q-TOF-MS technology," Chinese Journal of Experimental Traditional Medical Formulae, vol. 20, pp. 51-55, 2014.

[14] G. H. Zhao, C. P. Yan, Z. S. Xu, Q. Q. Gao, Z. P. Chen, and W. D. Li, "The effect of salt-processed Psoralea corylifolia on generative organ targeting," Journal of Analytical Chemistry, vol. 10, 2016.

[15] Y. Q. Huang, Y. N. Fan, T. Z. Jia, T. T. Geng, and J. Shi, "Effect of Morinda officinalis root and its salt-processing product on energy metabolism in rats with yang deficiency," Zhong Yao Cai, vol. 39, no. 5, pp. 1028-1031, 2016.

[16] J. Shi, N. Cui, and T. Z. Jia, "Effect of different processed products and extracts of Morinda officinalis root on adjuvantinduced arthritis in rats," Zhong Yao Cai, vol. 38, no. 8, pp. 1626-1629, 2015.

[17] N. Cui, J. Shi, H. Y. Jing, and T. Z. Jia, "Screening of effective fractions of Morindae officinalis Radix and its mechanism of action," Chinese Traditional Patent Medicine, vol. 35, no. 10, pp. 2256-2259, 2013.

[18] B. Song, F. Wang, and W. Wang, "Effect of aqueous extract from Morinda officinalis F.C. How on microwave-induced hypothalamic-pituitary-testis axis impairment in male sprague-dawley rats," Evidence-Based Complementary and Alternative Medicine, vol. 10, 2015.

[19] F. Wang, L. Wu, L. Li, and S. Chen, "Monotropein exerts protective effects against IL- $1 \beta$-induced apoptosis and catabolic responses on osteoarthritis chondrocytes," International Immunopharmacology, vol. 23, no. 2, pp. 575-580, 2014.

[20] Z. Zhang, Q. Zhang, H. Yang et al., "Monotropein isolated from the roots of Morinda officinalis increases osteoblastic bone formation and prevents bone loss in ovariectomized mice," Fitoterapia, vol. 110, pp. 166-172, 2016.

[21] L. Bao, L. Qin, L. Liu et al., "Anthraquinone Compounds from Morinda officina osteoclastic bone resorption in vitro," Chemico-Biological Interactions, vol. 15, no. 2-3, pp. 97-105, 2011.

[22] N. Cui, J. Shi, and T. Z. Jia, "Comparative study on kidney tonifying and yang supporting effects of different processed products of Morinda officinalis," Zhongguo Zhong Yao Za Zhi, vol. 38, no. 22, pp. 3898-3901, 2013.

[23] Y.-Q. He, Q. Zhang, Y. Shen et al., "Rubiadin-1-methyl ether from Morinda officinalis How. Inhibits osteoclastogenesis through blocking RANKL-induced NF-kB pathway," Biochemical and Biophysical Research Communications, vol. 506, no. 4, pp. 927-931, 2018.

[24] Q. Liu, S. B. Kim, J. H. Ahn, B. Y. Hwang, S. Y. Kim, and M. K. Lee, "Anthraquinones from Morinda officinalis roots enhance adipocyte differentiation in 3T3-L1 cells," Natural Product Research, vol. 26, no. 18, pp. 1750-1754, 2012.

[25] L. Chi, L. Chen, J. Zhang, J. Zhao, S. Li, and Y. Zheng, "Development and application of bio-sample quantification to evaluate stability and pharmacokinetics of inulin-type fructooligosaccharides from Morinda Officinalis," Journal of Pharmaceutical and Biomedical Analysis, vol. 156, pp. 125132, 2018.
[26] C. Li, J. Dong, J. Tian, Z. Deng, and X. Song, "LC/MS/MS determination and pharmacokinetic study of iridoid glycosides monotropein and deacetylasperulosidic acid isomers in rat plasma after oral administration ofMorinda officinalisextract," Biomedical Chromatography, vol. 30, no. 2, pp. 163-168, 2016.

[27] Y. Shen, Q. Zhang, Y. B. Wu et al., "Pharmacokinetics and tissue distribution of monotropein and deacetyl asperulosidic acid after oral administration of extracts from Morinda officinalis root in rats," BMC Complementary and Alternative Medicine, vol. 18, p. 288, 2018.

[28] Q. Zhou, H. Yan, R. Li, X. Li, and J. Wei, "Quantitative determination of monotropein in rat plasma and tissue by LCMS/MS and its application to Pharmacokinetic and tissue distribution studies," Revista Brasileira de Farmacognosia, vol. 28, no. 4, pp. 451-456, 2018. 